\title{
Patterned SLIPS for the Formation of Arrays of Biofilm Microclusters with Defined Geometries
}

\author{
Julia Bruchmann, Ivana Pini, Tejeshwar S. Gill, Thomas Schwartz,* and Pavel A. Levkin*
}

Biofilms represent an immense problem in medicine due to their strong drug-resistant properties and inherent stress-response activities. Due to the inhomogeneous and very complex architectures of large biofilm aggregates, biofilm studies often suffer from low reproducibility. In this study, an approach to form arrays of homogeneous biofilm microclusters with defined 2D geometries is presented. The method is based on the formation of water-infused hydrophilic porous polymer areas with precise geometries separated by "slippery" lubricant-infused porous surface (SLIPS). Due to the SLIPS' biofilm repellent properties, multiple identical 3D biofilm clusters are formed in the hydrophilic patches that can be used for biofilm screening. Formation of biofilm microcluster arrays of different bacterial strains of Pseudomonas aeruginosa on the SLIPS micropatterns is investigated. Critical parameters influencing minimal adhesive regions for biofilm attachment and minimal SLIPS dimensions to avoid biofilm adhesion are studied. The ability to produce arrays of biofilm microclusters with highly uniform, well-defined shapes opens an opportunity to study interactions of biofilms with various medically relevant factors with a better reproducibility and to investigate the complex biofilm architecture, heterogeneity, and interactions between biofilm subpopulations.

\section{Introduction}

Biofilms are accumulations of microbes embedded in a hydrated mixture of extracellular polymeric substances attached to a surface. ${ }^{[1]}$ Due to their ability to develop on various natural, technical, and medical surfaces, ${ }^{[2]}$ biofilms represent an immense problem in different applications. ${ }^{[3]}$ Biofilms are

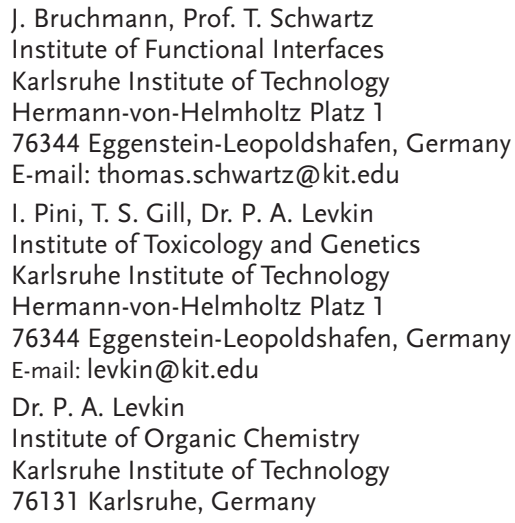

extremely resistant to both the immune response and systemic antibiotic treatments, and thus their development is the primary cause of, e.g., implant/catheterassociated infections. ${ }^{[4]}$

Bacterial infection on medical devices such as catheters and artificial prosthetics is a serious ongoing problem in the biomedical area. Of the 2.6 million orthopedic implants used annually only in the United States, $\approx 110000$ (4.3\%) lead to hospital acquired, or nosocomial, infections. ${ }^{[5]}$ When considering all implanted devices, the number of implant-related bacterial infections approaches one million per year. Another serious problem is that antibiotics administered systemically show constantly decreasing efficiency against implant-associated infections. ${ }^{[6]}$ These infections are caused by bacterial adhesion to an implant surface and by formation of bacterial biofilms.

Bacteria inside the biofilm cannot be considered as a simple sum of their constituent cells, but as complex differentiated communities with a heterogeneous 3D structure, ${ }^{[7]}$ which benefit from metabolic exchange and genetic flexibility. ${ }^{[8]}$ To some extent, biofilms can be compared with eukaryotic cell spheroids possessing complex $3 \mathrm{D}$ architectures and in vivo-like properties making them more relevant for drug screenings. ${ }^{\left[{ }^{9]}\right.}$ Thus, there is a strong need to develop methods that would allow for highthroughput screening of small 3D biofilm microclusters. Such biofilm aggregates should possess the same dimension and geometry in order to assure reproducibility and comparability. In addition, understanding of the complex biofilm organization, heterogeneity, and 3D architecture is crucial both for the development of advanced antifouling coatings and for utilizing unique biofilm properties in practical applications, ranging from biotechnology to diagnostics and tissue engineering.

A prerequisite for the development of a technology for highthroughput screening of biofilm aggregates and for studying biofilm organization and 3D architecture, however, requires inter alia methods to spatially control biofilm growth in arrays of precise 2D microstructures over several days. Due to the inherent properties of biofilms to adhere to almost any substrate, there are only very few approaches that allow for biofilm patterning and for making biofilm microarrays. ${ }^{[10]}$ When cultivation of biofilms over several days is required, the patterning 
ability becomes even more challenging due to the shortage of long-term biofilm-controlling coatings..$^{[1]}$

Here we report a method for creating arrays of precise micropatterns of biofilm clusters stable under defined biofilm culturing conditions for several days. First of all, this method opens the way to study and mimic complex biofilm architecture, heterogeneity, and interactions between biofilm subpopulations and between signaling factors. ${ }^{[12]}$ Second, this approach enables high-throughput screenings of arrays of homogeneous biofilm clusters with defined size and shapes. The method utilizes the ability to create micropatterns of slippery lubricant-infused porous surfaces (SLIPS), ${ }^{[13]}$ recently demonstrated to possess long-term biofilm resistant properties using hydrophilic-hydrophobic patterns formed in nanoporous polymer layers. ${ }^{[5 a, 11,14]}$ Three Pseudomonas aeruginosa strains with different capacities for biofilm formation were studied. ${ }^{[15]}$ The developed biofilm patterning method was used to investigate the effect of biofilm bridging between separated islands of biofilms. The dependence of biofilm growth and biofilm-resistance on the size of SLIPS regions was investigated.

\section{Results and Discussion}

2D patterns of a thin perfluoropolyether (Krytox) layer impregnating the hydrophobic porous polymer substrate were created by forming a hydrophilic-hydrophobic micropattern in porous poly(2-hydroxyethyl methacrylate-co-ethylene dimethacrylate) (HEMA-EDMA) film. The polymer substrate was synthesized by UV-initiated free-radical polymerization on a glass substrate according to a previously published procedure and functionalized using the UV-induced thiol-yne reaction between the alkyne functionalities in the polymer and the thiol-groups of perfluorodecanethiol (hydrophobic region) or cysteamine$\mathrm{HCl}$ (hydrophilic region) to generate hydrophilic-hydrophobic micropatterns of defined sizes (Table 1). ${ }^{[16]}$ The thickness of the porous polymer layer was $15 \mu \mathrm{m}$ with pores of $100-500 \mathrm{~nm}$, according to scanning electron micrographs (Figure S1, Supporting Information).

The hydrophilic areas showed highly wettable properties with apparent static water contact angle (WCA) of $11^{\circ} \pm 2^{\circ}$. The fluorinated hydrophobic regions of the porous HEMA-EDMA layer demonstrated a static WCA of $135^{\circ} \pm 2^{\circ}$, making these regions nonwettable for aqueous solutions but at the same time highly oleophilic.

In order to create a pattern of the SLIPS regions, the hydrophilic-hydrophobic polymer substrate was dipped into an aqueous solution, resulting in the formation of an array of

Table 1. Geometries and sizes of hydrophobic-hydrophilic patterns.

\begin{tabular}{lcc}
\hline Pattern geometry & $\begin{array}{c}\text { Pattern size } \\
{[\mu \mathrm{m}]}\end{array}$ & $\begin{array}{c}\text { Distance between hydrophilic } \\
\text { regions }[\mu \mathrm{m}]\end{array}$ \\
\hline Square & 350 (edge) & 100 \\
Triangle & 300 (edge) & 60 \\
"Drop" & 300 (internal diameter) & 300 \\
Line & $10,15,20,30,50,75,100,250$, & 250 \\
& 500,1000 (width) & \\
\hline
\end{tabular}

separated microdroplets in every hydrophilic spot surrounded by dry hydrophobic porous regions (Figure 1, Step 1). ${ }^{[17]}$ In the next step the array of aqueous microdroplets was carefully covered with Krytox lubricant (Figure 1, Step 2). Krytox only infuses the nonwettable porous hydrophobic areas, covering the array of water droplets immobilized in the hydrophilic spots. The excess of Krytox is then removed by dipping the substrate into water for several times, leading to the formation of a binary pattern of lubricant-infused SLIPS, replicating the geometry of the hydrophobic pattern, and water-infused porous hydrophilic regions (Figure 1, Step 2).

The apparent static WCA on the SLIPS regions is $96^{\circ} \pm 2^{\circ}$. The static contact angle for chloroform is $\approx 50^{\circ}$. Both liquids' sliding angles are close to $0^{\circ}$, indicating the "slippery" behavior and the repellency toward different solvents. ${ }^{[11]}$

\subsection{Influence of Surface Wettability and Krytox Application on Biofilm Growth}

Having characterized the surface properties, hydrophilic porous polymer, hydrophobic porous polymer, and SLIPS were tested for biofilm growth. Previously, we demonstrated that both superhydrophobic and SLIPS properties could successfully repel adhesion of eukaryotic HeLa cells for more than $7 \mathrm{~d} .{ }^{[16 a, 17]}$ The biofilm repellent property of the SLIPS was also demonstrated. ${ }^{[11]}$ Here we investigate the ability to direct biofilm growth using hydrophobic-hydrophilic as well as SLIPShydrophilic micropatterns. Thus, biofilms of $P$. aeruginosa strain PA01, which is a commonly used laboratory strain, were grown for $3 \mathrm{~d}$ on both types of patterns (air- and lubricantinfused; see Figure 1, pattern after step 1 and pattern after step 2). Biofilms were stained with 5-cyano-2,3-ditolyltetrazolium chloride (CTC) and the surfaces were analyzed by fluorescence microscopy with subsequent software mediated image analysis (Figure 2A,B).

Binary images of biofilms on triangular hydrophilic patterns with hydrophobic borders (Figure 2A) showed a consistent coverage of bacterial cells in both hydrophilic and hydrophobic areas. The coverage of the hydrophobic and hydrophilic regions was $2.2 \%$ and $2.6 \%$, respectively (Figure $2 \mathrm{~B}$ ). On the contrary only $0.014 \%$ of the SLIPS region is occupied by biofilm, keeping the same $2.6 \%$ occupation for the hydrophilic regions next to the SLIPS regions (Figure 2B). Thus, the SLIPS region is occupied by the biofilm 185 times less than the neighboring hydrophilic regions on the same surface under exactly the same conditions. The hydrophobic regions noninfused with Krytox are occupied only 1.2 times less than the neighboring hydrophilic water-infused regions, demonstrating the biofilm repellant properties of the SLIPS.

It is important to mention that substrates possessing adjacent regions of different properties (e.g., hydrophilichydrophobic, hydrophilic-SLIPS, etc.) enable perfect parallel experiments for studying the difference in cell-surface interactions and the influence of different surface properties on cellular behavior, such as biofilm adhesion or repellency. The use of micropatterned substrates allows us to study cell-surface interactions under exactly the same conditions. 


\section{STEP 1: Surface patterning}

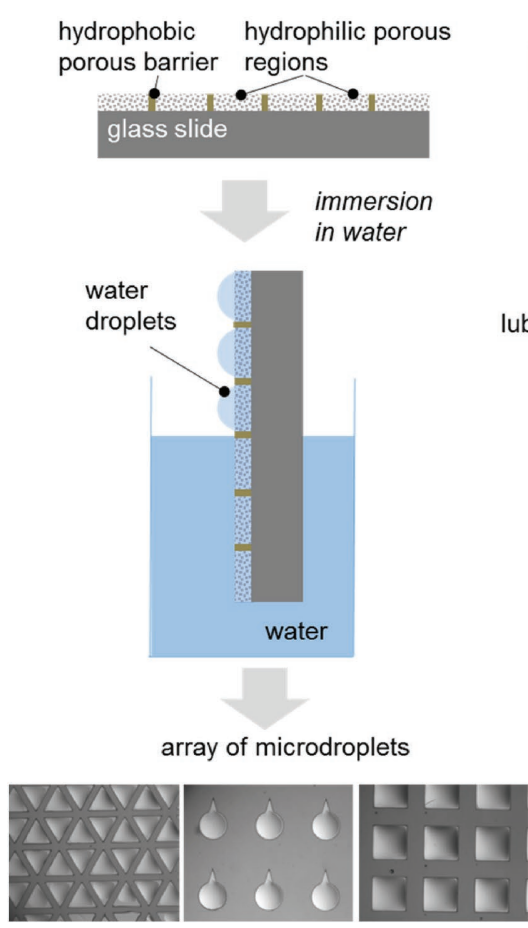

STEP 2: Application of lubricant

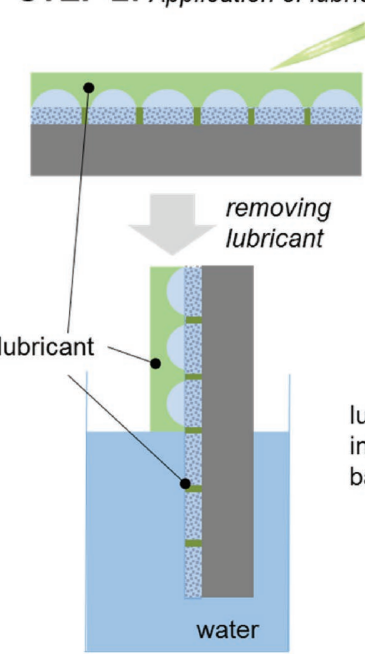

immersion in culture medium

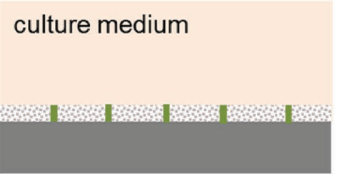

STEP 3: Seeding of bacteria and biofilm formation

Figure 1. Formation of patterned SLIPS. Step 1: When the hydrophilic-hydrophobic patterned substrate is immersed in water, an array of microdroplets is formed on the hydrophilic areas while the hydrophobic areas remain dry (effect of discontinuous dewetting). Different geometries of microdroplet arrays are shown (dimensions see Table 1). Step 2: A thin layer of Krytox is applied over the substrate infusing only the nonwetted hydrophobic areas of the porous polymer, forming a SLIP surface. The surface is dipped first into water to wash off the excess of Krytox covering the water droplets and then into culture medium to replace water with medium. Step 3: Bacteria cultured on the substrate adhere to the hydrophilic areas but are repelled by the SLIPS regions. Step 4: Fluorescence staining and image analysis of $P$. aeruginosa biofilm on hydrophilic squares separated by SLIPS barriers.

\subsection{Comparison of Biofilm Occupation on Hydrophilic, Hydrophobic, and SLIPS Regions}

After this preliminary study performed on PA01 strain using triangular hydrophilic patterns, we extended the study of hydrophilic-SLIPS micropatterns to other P. aeruginosa strains and to other geometries and sizes of hydrophilic areas, in order to analyze and compare the biofilm growth and ability to biofilm clusters of defined geometry through the SLIPS patterns. Indeed, biofilm formation events including adhesion to surfaces, biofilm growth, development of 3D structures, and dispersal are depending on the investigated bacterial strain and can vary among a species. ${ }^{[18]}$ Thus we used PA01, which has a low biofilm formation capacity and is highly sensitive against antibiotics and PA30 and PA49, which are environmental wastewater isolates. ${ }^{[19]}$ In particular, PA30 is an antibiotic sensitive strain with intermediate biofilm forming capacity and PA49 is an antibiotic multiresistant strain with very high biofilm formation capacity. ${ }^{[19]}$ All three strains were incubated on the patterned SLIPS for $3 \mathrm{~d}$ under static conditions, followed by staining with CTC and quantification using fluorescence microscopy with subsequent image analysis (Figure 2C).

Biofilm formation was in all cases much higher in the hydrophilic areas compared to the SLIPS regions (Figure 2C). Strain PA01 showed only weak biofilm formation with $4.7( \pm 6.4) \%$ occupation of the hydrophilic regions and only $0.01( \pm 0.6) \%$ of SLIPS (Figure 2C). Strain PA30, an intermediate biofilm former, showed $21.8( \pm 19.6) \%$ occupation of the hydrophilic areas and only $0.1( \pm 0.8) \%$ in the SLIPS regions (Figure $2 \mathrm{C}$ ). The strongest biofilm forming strain PA49 showed the highest biofilm occupation values with $38.8( \pm 17.2) \%$ in the hydrophilic area and $10.3( \pm 9.9) \%$ on SLIPS (Figure 2C). The indicated standard deviations might result from different physiological activities during inoculation phases due to natural variations of biological systems and the fact that bacterial suspensions are not synchronized in growth. As mentioned, the cell densities were kept constant at the inoculation (see the Experimental Section). Nevertheless, for all strains a significant difference between the hydrophilic and SLIPS regions was observed $(p \leq 0.001)$, being $p_{\mathrm{PA} 01}=5.8 \times 10^{-7}, p_{\mathrm{PA} 30}=5.3 \times 10^{-8}$, and $p_{\text {PA49 }}=6.1 \times 10^{-13}$. The highest occupation of the SLIPS area was observed for strain PA49, which confirmed our previous observation on a nonpatterned SLIPS. ${ }^{[11 b]}$ Thus, the difference in the biofouling of the hydrophilic versus SLIPS regions ranged from 470 times for PA01 to 3.8 times for PA49.

\subsection{Guiding Biofilm Formation Using SLIPS Micropatterns}

To further evaluate the ability to pattern biofilms, SLIPS patterns were prepared with different geometries (Table 1). Triangular, squared, and drop shaped hydrophilic regions 


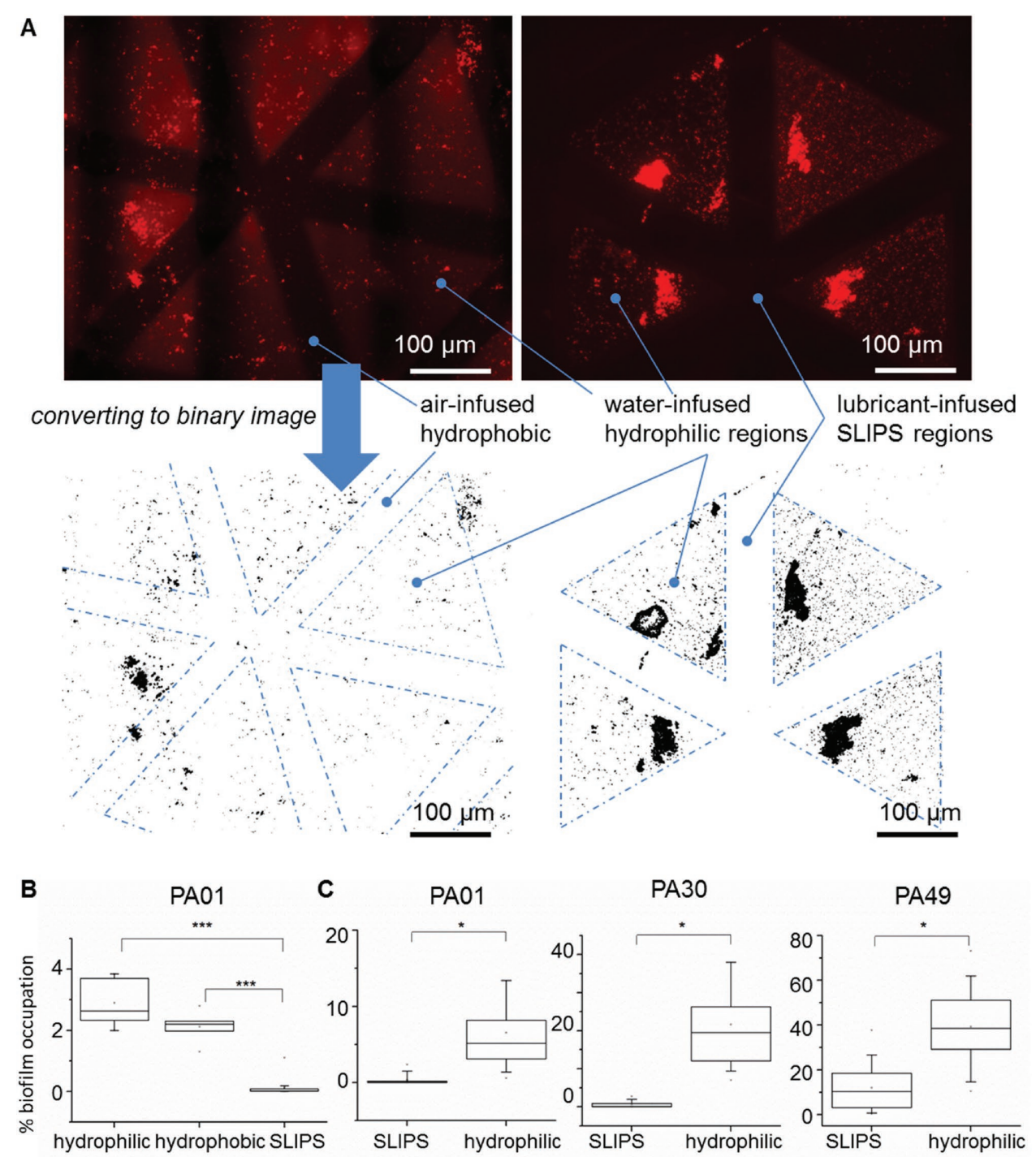

Figure 2. Influence of surface properties on biofilm formation. A) Fluorescence microscope images of $P$. aeruginosa (PA01 (A)-(C); PA30 (C); and PA49 (C)) grown on both hydrophilic-air-infused hydrophobic (left) and SLIPS-hydrophilic patterns (right). Biofilms were stained after $3 \mathrm{~d}$ and binary images were produced. B) Quantification of biofilm occupation in different areas corresponding to the hydrophilic-air-infused hydrophobic patterns with a comparison to the biofilm occupation on the SLIPS area. C) Quantifications of the biofilm occupation (different bacteria strains) on the SLIPShydrophilic patterns. ***'t-test: $p \leq 0.001, \alpha=0.05 ; * t$-test: $p \leq 0.001, \alpha=0.01$.

(water-infused) surrounded by SLIPS regions (lubricantinfused) were formed. In addition, inverse geometries with hydrophilic background and SLIPS occupying spots of different shapes were also prepared and tested with the three different bacteria strains: PA01, PA30, and PA49. Patterns were incubated with bacteria for $3 \mathrm{~d}$ under static conditions, stained and imaged with fluorescence microscopy. Figure 3 demonstrates the ability to control biofilm formation spatially for all three bacterial strains.

Biofilm occupation of both hydrophilic and SLIPS regions increased in the order PA01 < PA30 < PA49. Biofilm formation by PA49 was different and first occupied the hydrophilic regions almost to $100 \%$ and then spread even to the SLIPS regions. This was observed for all different geometries including the inverse patterns. It becomes obvious that the size and the geometry of the SLIPS area have a significant effect on biofilm growth. In large hydrophilic areas interrupted by small SLIPS areas, strains PA30 and PA49 are able to colonize the edges of the hydrophobic pattern or even span networks over the hydrophobic structures, especially in drop-shaped and triangular patterns (Figure 3, red arrows).

In some cases even the whole small SLIPS regions could be covered by a biofilm network (Figure 4A). These bridges were further analyzed by taking 3D images using fluorescence microscopy (Figures 4 and 5). These images show that the biomass covering the SLIPS area connected to biofilms attached to the hydrophilic area (Figure 4A). The fluorescence 3D images (Figure 4A) also indicate the presence of the Krytox superficial layer covering the hydrophobic spots with biofilm above it. 


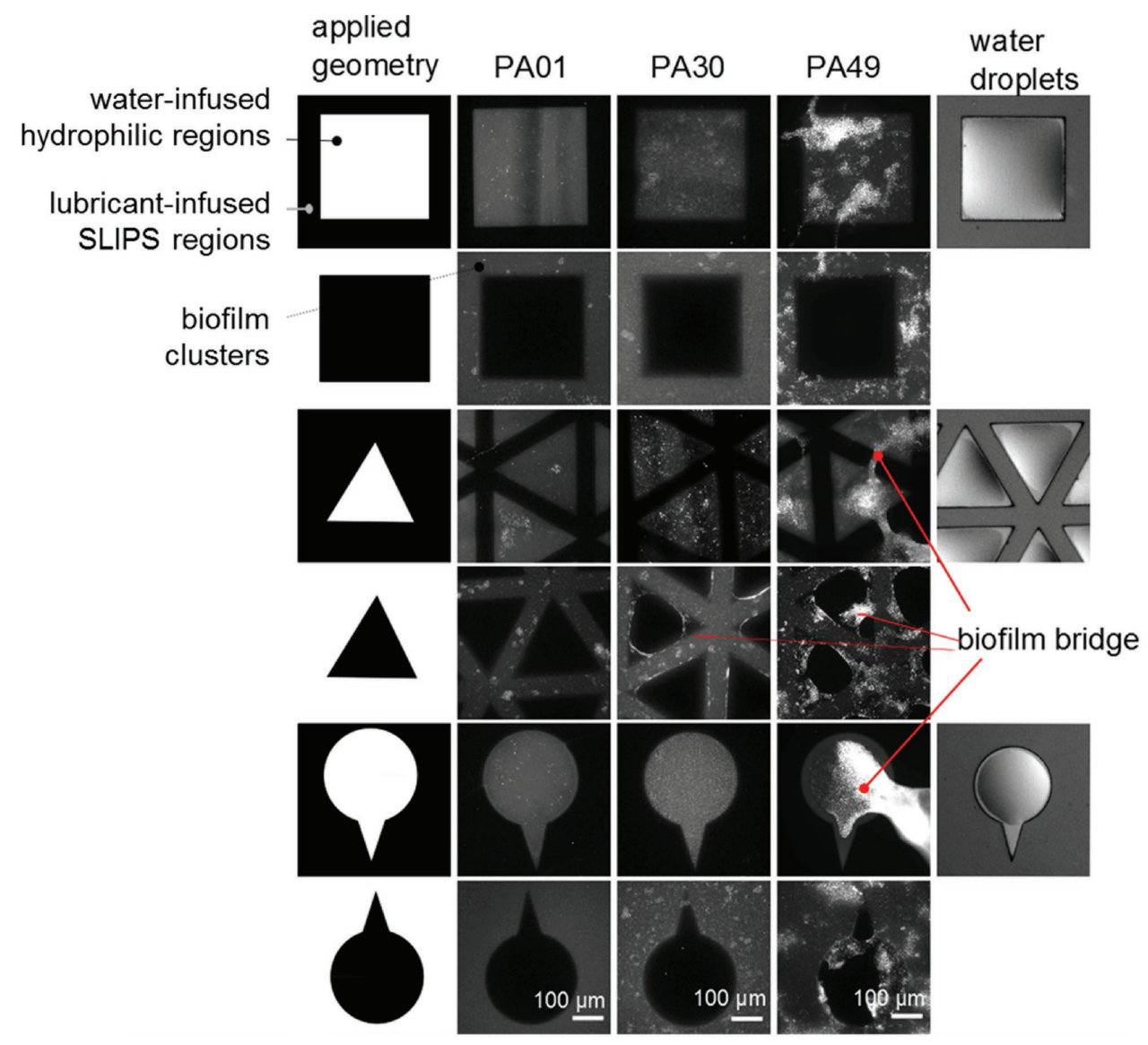

Figure 3. Fluorescence microscopy images of biofilms formed on hydrophilic-SLIPS micropatterns. P. aeruginosa strains PA01, PA30, and PA49 were used to form biofilms using $3 \mathrm{~d}$ static culture in BM2 medium. Biofilms were visualized by CTC staining. Right: Water droplets formed in the hydrophilic area after the initial water application. Red arrows indicate biofilms bridging biofilm repellent SLIPS areas.

For larger hydrophobic SLIPS areas and smaller hydrophilic areas the same phenomenon was observed (Figure 4B). Biofilm attachment points were only visible in the hydrophilic regions of the substrate (Figure 4B, bottom view). The biofilm in the hydrophobic area was located above the polymer surface and was connected to the biofilm in the hydrophilic pattern via thin bridges (Figure 4B, top view). For strains PA01 and PA30 no bridge formation was observed. In a previous publication we have demonstrated that the multiresistant $P$. aeruginosa strain PA49 was significantly different in the RAPD-based analyses of the genome patterning compared to the other used $P$. aeruginosa strains. ${ }^{[11 \mathrm{~b}]}$ Still it is unknown which strains' specific factor is responsible for the bridge formation. The biofilm bridging formation for $P$. aeruginosa biofilms has been observed previously in the low $\mu \mathrm{m}$ range. Kappell et al. showed bacterial bridges across gaps of about $1 \mu \mathrm{m}$ widths between patterns imprinted into silicone elastomers. ${ }^{[10 f]}$ The ability to overcome small distances was observed also for other kinds of bacteria biofilms. For example, E. coli grown on gold surfaces modified with square patterns of self-assembled monolayers could build bridges between patterns of cell clusters separated by distances less than $10 \mu \mathrm{m} \cdot{ }^{[10 \mathrm{~d}]} \mathrm{How}$ ever, biofilm bridges over larger distances (here $300 \mu \mathrm{m}$ ) have not been demonstrated to the best of our knowledge. By monitoring the biofilm development in real time, the authors found that these connections among clusters were formed during biofilm maturation and not during the early stage of nonspecific binding of seeding cells, which proved that clusters interaction was correlated to growth and division of cells belonging to clusters close to each other. ${ }^{[10 \mathrm{~d}]}$ Therefore, the size of the clusters and distance between them could influence interactions between cell clusters.

\subsection{Conditions for Bacterial Bridging}

For the control of biofilm-drug interaction on biofilm patterned surfaces, bridging of biofilm repulsive areas should be avoided especially in high-throughput experiments analyzing a large number of distinct biofilm spots. Therefore, conditions in surface design should be identified to avoid the bridging behavior. Since connections between bacterial clusters seem to be dependent on size of the clusters and distance between each other, ${ }^{[10 \mathrm{~d}]}$ in this study the minimal size of hydrophilic spots necessary for biofilm formation was further explored. Here, the dependence from the distance between biofilm clusters and the ability to form bridges between them was analyzed.

Stripe-shaped patterns having a gradient of sizes of hydrophilic and SLIPS areas ranging from 10 to $1000 \mu \mathrm{m}$ were created (Figure 5). These surfaces of hydrophilic and SLIPS 

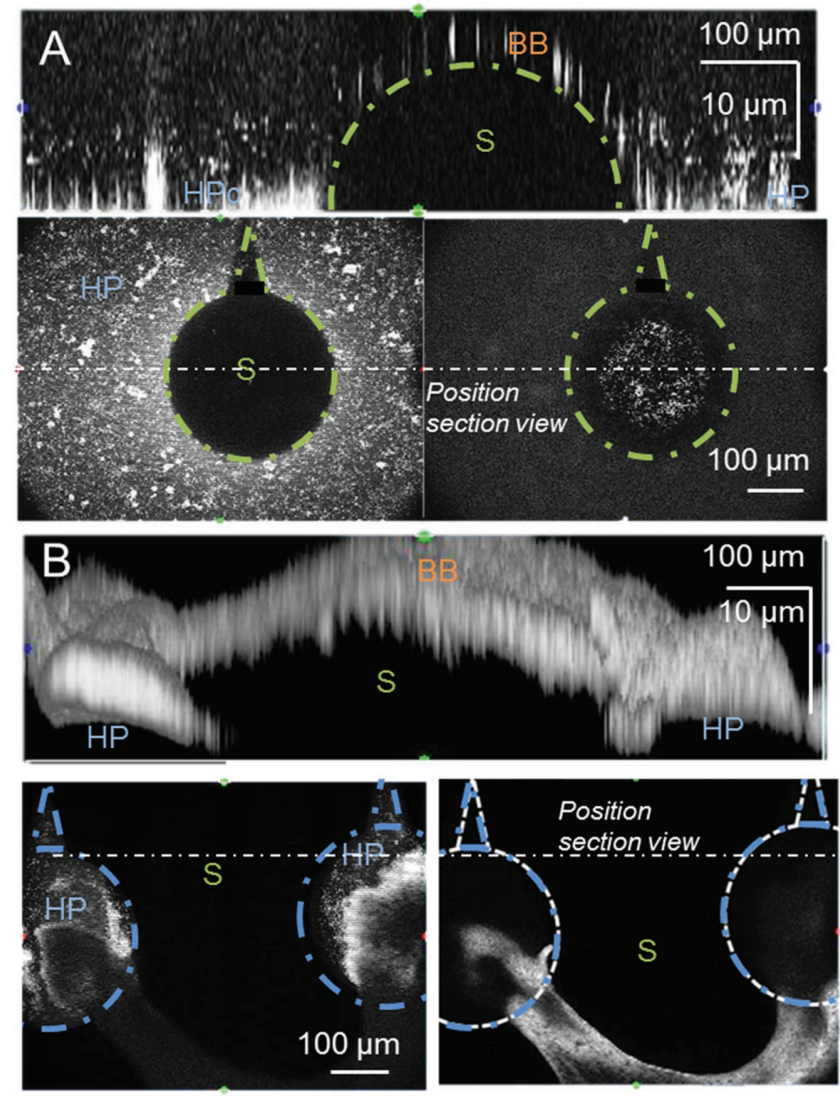

biofilm bridge BB SLIPS S hydrophilic water-infused polymer HP

Figure 4. Biofilm bridge formation. P. aeruginosa biofilm (strain PA49) is grown on a A) SLIPS pattern or B) inverse pattern for $3 d$ in static Petri dishes in BM2 medium. Staining is performed using CTC. A section view, the bottom layer, and the top layer of the biofilm are shown. Green and blue dotted lines indicate the position of the patterned geometry. (A): SLIPS, (B): hydrophilic.

stripes were again incubated with the three different bacterial strains of $P$. aeruginosa (PA01, PA30, and PA49) for $3 \mathrm{~d}$ according to the previously described experiments.

In the case of the weak biofilm formers, PA01 and PA30, SLIPS stripes smaller or equal to $30 \mu \mathrm{m}$ width did not interrupt biofilm formation yielding to continuous biofilm. Above $50 \mu \mathrm{m}$ hydrophilic stripe width clearly separated biofilm clusters were formed. Regarding the minimal size of hydrophilic area necessary for bacterial attachment, biofilm formation was observed from $30 \mu \mathrm{m}$ wide stripes for PA01 and from $75 \mu \mathrm{m}$ wide stripes for PA30. In smaller areas no or only single bacterial cell attachment was observed (data not shown).

A different behavior was found for the stronger biofilm former strain PA49 (Figure 5; Figure S2, Supporting Information). In case of small hydrophilic patterns $(A=10-30 \mu \mathrm{m})$ separated by SLIPS of a constant width $(\mathrm{B}=250 \mu \mathrm{m})$, no biofilm formation occurred and only single cell attachment was observed (Figure 5A,C; Figure S2a, Supporting Information), indicating a minimal pattern size of $50 \mu \mathrm{m}$ necessary for biofilm formation. In the second scenario of intermediate hydrophilic pattern sizes $(A=50-250 \mu \mathrm{m})$, hydrophilic lines were occupied by $40 \%$ (Figure 5A,C; Figure S2b, Supporting Information). Furthermore, bacterial bridges spanning over the SLIPS areas connecting the hydrophilic clusters were observed. In the third scenario with large hydrophilic areas $(A=250-500 \mu \mathrm{m})$ still a high occupation of the hydrophilic area was detected, but no bacterial biofilm bridges were observed (Figure S2c, Supporting Information). This suggests that the biofilm cluster size is important for bacterial bridge formation.

Varying the SLIPS sizes ( $\mathrm{B}=15-1000 \mu \mathrm{m}$ ) between hydrophilic lines of constant widths $(A=250 \mu \mathrm{m})$ (Figure 5B,D; Figure S2d, Supporting Information), full biofilm occupation in all hydrophilic areas as well as in small SLIPS areas $(B=15-20 \mu \mathrm{m})$ was observed. Biofilm growth was not interrupted by the SLIPS lines narrower than $20 \mu \mathrm{m}$. In case of SLIPS of intermediate width $(30-100 \mu \mathrm{m})$, biofilm bridges between the hydrophilic patterns were observed. SLIPS lines wider than $250 \mu \mathrm{m}(250-1000 \mu \mathrm{m})$ completely prevented biofilm bridging. This suggests that the distance between the biofilm clusters is important for the formation of biofilm bridges.

The results above demonstrate that bacterial density, size of the hydrophilic pattern, and width of SLIPS regions are important for biofilm patterning and formation of biofilm bridges. There might be a factor we call "tension to spread," which causes biofilm bridging in case of intermediately sized hydrophilic clusters but not between large hydrophilic clusters. This factor called here "tension to spread" can be compared to the streamer formation in biofilm under fluidic flow conditions, where also fine filamentous structures promote biofilm spreading. ${ }^{[20]}$ We suppose that all these observed factors act cooperatively, influencing each other.

There were differences observed in biofilm bridge formation and pattern coverage among different $P$. aeruginosa strains. Genome analyses of the $P$. aeruginosa strains PA49, PA30, and PA01 demonstrated that large fractions of the genomes belong to the highly conserved core genome containing only few highly variable regions, while most of the genetic variation between species is restricted to the so-called accessory genome organized in various regions of genomic plasticity. ${ }^{[15]}$ Investigating the transcriptional level between the strains, also here differences were observed by usage of different matrices or stress factors. Thus, these genome analyses demonstrated that the variable regions of accessory genome together with transcriptional regulations in $P$. aeruginosa might play an important role in strain specific adaptation to specific environments including biofilm formation on SLIPS patterned surfaces. Still, specific genes responsible for spreading behavior are not identified.

Here, differences among $P$. aeruginosa isolates were shown. Regarding other hygienically relevant microbes, we also expect differences in biofilm formation and behavior. This assay, with easily adaptable parameters of pattern geometries, enables customizable production of homogenous biofilm patterns of all kinds of relevant biofilms by determination of critical parameters for each investigated microbe.

\section{Conclusion}

In this study we demonstrate a novel technique based on patterned SLIPS for creating arrays of biofilm microclusters with 

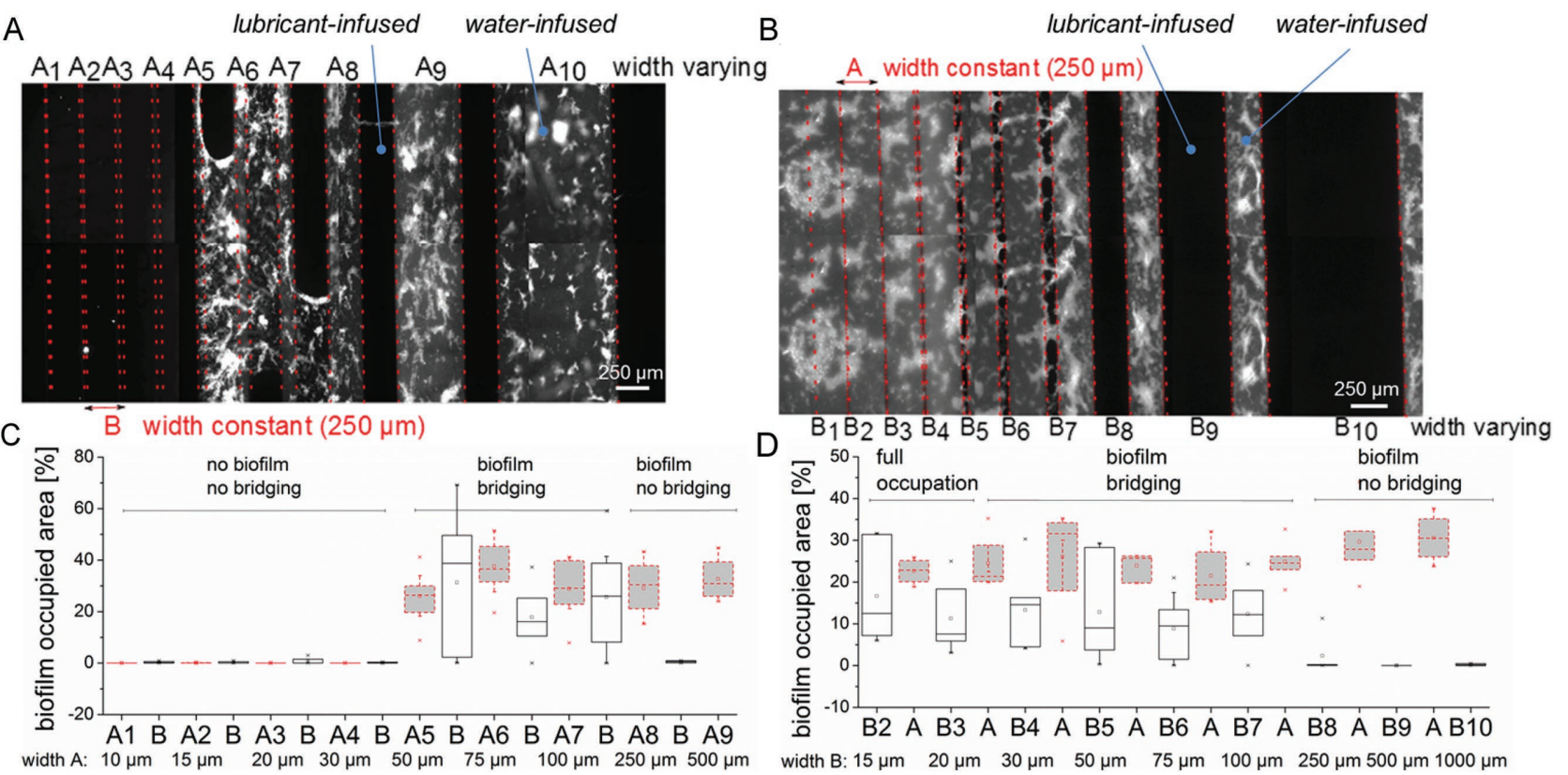

Figure 5. Biofilm bridge formation on patterned surfaces with varying sizes of water-infused hydrophilic and lubricant-infused SLIPS areas. $P$. aeruginosa strain PA49 was grown for $3 \mathrm{~d}$ on the patterned surfaces with varying widths of hydrophilic regions $((A, C)$ grey bars) or of hydrophobic regions ((B,D) white bars) and afterward stained with CTC (width dimensions are listed in (C,D)). Biofilm occupied area was calculated by image analysis (C,D).

precise geometry and stability over several days. Three different wild-type strains of $P$. aeruginosa were investigated on various micropatterns. The results demonstrated that all strains formed biofilm microclusters with defined geometries restricted to the hydrophilic regions. The size of SLIPS and hydrophilic areas appear, however, to influence the biofilm formation of different $P$. aeruginosa strains. An interesting phenomenon of biofilm bridging was observed when the strong biofilm-former $P$. aeruginosa (PA49) was grown on patterned SLIPS regions with the width less than $250 \mu \mathrm{m}$ or hydrophilic regions between 50 and $250 \mu \mathrm{m}$, suggesting that biofilms can occupy even completely biofilm resistant surfaces provided there are enough anchorage points available. Biofilm bridging also depended on cellular densities in the biofilm clusters and was not observed in the case of low and intermediate biofilm producing strains PA01 and PA30.

The ability to produce arrays of biofilm microclusters, which are equally sized and possess the same and controllable geometries stable over several days, opens a unique opportunity to study interactions of biofilms with various factors including drugs or host immune system molecules in a high-throughput way with better reproducibility. ${ }^{[12]}$ Such arrays of identical biofilm microclusters are important for obtaining more reliable drug response results due to the inherent complex biofilm architecture and heterogeneity of large biofilms formed in standard microtiter plates, Petri-dishes, or flow cells. In addition, the ability to control the shape and size of biofilm architectures as well as distance between the adjacent biofilm clusters is crucial for the fundamental investigation of the complex biofilm architecture, heterogeneity, and interactions between biofilm subpopulations.

\section{Experimental Section}

Materials: Sodium hydroxide, hydrochloric acid, 1-decanol, 4-(dimethylamino)pyridine (DMAP), ethanol, acetone, dichloromethane, iron sulfate, and glucose were purchased from Merck (Darmstadt, Germany). Trichloro $(1 \mathrm{H}, 1 \mathrm{H}, 2 \mathrm{H}, 2 \mathrm{H}$-perfluorooctyl)silane, 3-(trimethoxysilyl)propyl methacrylate, HEMA, EDMA, cyclohexanol, 2,2-dimethoxy-2-phenylacetophenone, $\quad 1 \mathrm{H}, 1 \mathrm{H}, 2 \mathrm{H}, 2 \mathrm{H}$ perfluorodecanethiol, magnesium acetate, and Tris-base were bought from Sigma-Aldrich (Steinheim, Germany). 4-Pentynoic acid was accessed from Apollo Scientific (Manchester, England). N, $N^{\prime}$ diisopropylcarbodiimide was acquired from Alfa Aesar (Karlsruhe, Germany), cysteamine hydrochloride from AppliChem (Darmstadt, Germany), Krytox GPL 103 (Dupont KrytoxR GPL 103) from H Costenoble $\mathrm{GmbH} \&$ Co. KG (Eschborn, Germany), and CTC from Polysciences Europe $\mathrm{GmbH}$ (Eppelheim, Germany). Glass slides (Nexterion glass B) used as substrates to create the hydrophilic-hydrophobic pattern were purchased from Schott (Mainz, Germany).

Instruments: Deep UV collimated light source (OAI Model 0130-04706, San Jose, CA, USA) with 500 W Hg-xenon lamp (USHIO, Japan) and UV power meter (OAI 360) with $260 \mathrm{~nm}$ probe head UK1117 digital camera (EHD Imaging $\mathrm{GmbH}$, Damme, Germany) was used to take images of water droplets for water contact angle measurements. A modular pump (Harvard Apparatus, Hugstetten, Germany) was used for measurements of dynamic contact angles. Astereomicroscope Leica MZ10F (Leica, Wetziar, Germany) with Digital Camera DFC360FX (Leica, Wetziar, Germany) was used for images of wetted surfaces and a Axioplan 2 imaging system (Carl Zeiss, Oberkochen, Germany) equipped with AxioCam MRm camera and the AxioVision 4.6 software and an ApoTome was used for fluorescence microscopy. Scanning electron microscopy (SEM, Zeiss, Germany) for images of the polymer surface was used at $10.00 \mathrm{kV}, 3.4 \mathrm{~mm}$ working distance, with InLens detector. Samples were sputtered with $15 \mathrm{~nm}$ thick gold layer before the SEM analysis.

Glass Slides Activation and Modification: Activation of glass slides was done by dipping the slides in $1 \mathrm{M} \mathrm{NaOH}$ for 30 min and then in $1 \mathrm{M} \mathrm{HCl}$ 
for $1 \mathrm{~h}$ in order to create hydroxilic groups on the glass surface. Slides were then washed with deionized water and modified with $70 \mu \mathrm{L}$ of $20 \%$ 3-(trimethoxysilyl)propyl methacrylate solution in ethanol (solution was applied two times for $30 \mathrm{~min}$ ) to have exposed methacrylate groups on the surface.

Polymerization and Esterification of Modified Glass Slides: Previously modified glass slides were coated with $60 \mu \mathrm{L}$ prepolymerization solution containing monomer (2-hydroxyethyl methacrylate, 24 wt\%), crosslinker (ethylene dimethacrylate, $16 \mathrm{wt} \%$ ), porogens (mixture of cyclohexano and $n$-decanol 4:1 w/w, $60 \mathrm{wt} \%$ ), and the initiator (2,2-dimethoxy-2phenylacetophenone, $1 \mathrm{wt} \%$ with respect to the methacrylates). ${ }^{[16 a]}$ The polymerization reaction on the slide occurred using UV lamp for $15 \mathrm{~min}$ at $10 \mathrm{~mW} \mathrm{~cm}{ }^{-2}$ intensity and $260 \mathrm{~nm}$ wavelength. The intensity of UV light was calibrated using UV power meter (OAI 360) with $260 \mathrm{~nm}$ probe head. A nanoporous polymeric layer of HEMA-EDMA formed on the surface; its thickness was controlled at $15 \mu \mathrm{m}$ by the use of silica bead spacers of the corresponding diameter before applying the polymerization mixture. Slides to be used for biofilm experiments without Krytox GPL 103 infusion were taped with Tesa (Scotch tape) while those used for experiments with Krytox application were not taped to keep the surface more flat. The absence of the microroughness on the surface introduced with taping leads to lower hydrophobicity (absence of superhydrophobicity). Two HEMA-EDMA surfaces were then esterified simultaneously using $50 \mathrm{~mL}$ cold $\left(-20^{\circ} \mathrm{C}\right)$ dichloromethane solution of 4-pentynoic acid (111.6 mg), 4-dimethylaminocarbodiimide (56 mg) as catalyst, and $N, N^{\prime}$-diisopropylcarbodiimide $(180 \mu \mathrm{L})$ as coupling reagent, in order to have reactive alkyne functional groups at the surface. Slides were kept in the solution for $4 \mathrm{~h}$ in stirring conditions.

Patterning of Polymer Layers Prepared on Glass Slides via Thiol-Yne Reaction: The alkyne-modified porous polymer surface was functionalized via UV-induced thiol-yne click reaction with thiols and, depending on the properties of the thiol used, a hydrophobic or a hydrophilic surface could be obtained. ${ }^{[16 b]}$ A hydrophobic-hydrophilic array was created by modifying the substrate first with $1 \mathrm{H}, 1 \mathrm{H}, 2 \mathrm{H}, 2 \mathrm{H}$-perfluorodecanethio (5\% v/v solution in acetone) $\left(10 \mathrm{~mW} \mathrm{~cm}{ }^{-2}\right.$ UV light measured at $260 \mathrm{~nm}, \mathrm{Xe}-\mathrm{Hg}$ bulb, irradiation time $60 \mathrm{~s})$ through a quartz photomask to create hydrophobic regions where the UV-light could pass through the photomask and then with 15 wt\% cysteamine hydrochloride in 1:1 solution of ethanol and water through a quartz-slide to create hydrophilic regions by UV-initiated reaction with the nonreacted alkyne groups. Depending on the patterns of the photomask used, different shapes and geometries of hydrophilic as well as hydrophobic regions could be created (Table 1). Patterns with inverse hydrophobicity and hydrophilicity were produced by inverting the order of the use of the hydrophilic and the hydrophobic reagents.

SLIPS Preparation: To produce SLIPS, the patterned hydrophobichydrophilic polymer surface was first sterilized with $70 \%$ ethanol, then dried and dipped into water to form an array of droplets in all hydrophilic regions separated by dry hydrophobic regions (Figure 1, Step 1). Then, a thin layer of a perfluoropolyether liquid, Krytox GPL 103, was spread over the surface to cover both hydrophobic regions and water droplets (Figure 1, Step 2). The perfluoropolyether liquid (Krytox) only penetrates the pores of the hydrophobic areas; the excess of Krytox liquid that covered the droplet array was removed by dipping the substrate into water for 20 times.

Biofilm Formation on SLIPS: To study biofilm formation on patterned SLIPS, liquid cultures of $P$. aeruginosa strains PA01,[21] PA30, and PA49 ${ }^{[22}$ were inoculated in basal medium $2\left(\mathrm{BM} 2 ; 62 \times 10^{-3} \mathrm{M}\right.$ potassium

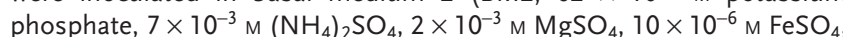
and $0.4 \%$ glucose $)^{[23]}$ and incubated at $37^{\circ} \mathrm{C}$ and $150 \mathrm{rpm}$ overnight. Bacterial cultures were adjusted to an optical density of $600 \mathrm{~nm}\left(\mathrm{OD}_{600}\right)$ of $0.1 \quad \approx 1 \times 10^{8}$ bacteria per $\left.\mathrm{mL}\right)$ and inoculated into petri dishes containing patterned SLIPS substrates and incubated for $3 \mathrm{~d}$ at room temperature with $40 \mathrm{rpm}$ shaking (Figure 1, Step 3). The medium was refreshed every $24 \mathrm{~h}$.

CTC Viability Staining: After $3 \mathrm{~d}$ of biofilm growth substrates were removed from the petri dishes, washed once with buffer $\left(5 \times 10^{-3} \mathrm{M}\right.$ magnesium acetate, $10 \times 10^{-3} \mathrm{M}$ Tris-base, $\mathrm{pH} 8$ ) and then placed into a solution of CTC $\left(4 \times 10^{-3} \mathrm{M}\right)$ in BM2 for $3 \mathrm{~h}$ at room temperature under gentle shaking in the dark. CTC is converted by metabolically active cells into red fluorescent molecule CTC-formazan. Afterward slides were again washed by dipping into Tris-buffer.

Fluorescence Microscopy: The stained biofilms were analyzed by epifluorescence microscopy with 100- and 200-fold magnification using Axioplan 2 imaging system with the filter set BP546/12; FT 580; LP 590 for CTC. Digital images and Z-stacks of the samples were obtained. At least three images per sample were acquired in both magnifications. The fluorescence microscopy was performed under air.

Biofilm Quantification: To compare biofilm occupation of hydrophilic and hydrophobic areas with and without Krytox, image analysis using Image J was performed. Binary images were produced using Image) option for individual thresholding, where all pixels above the threshold intensity appear black, the lower intensity pixels white. Threshold was adjusted for the visible biofilm, not for the background staining of the hydrophilic polymer. The biofilm occupied area was calculated from the black part of the image. At least 3 images were analyzed for each sample.

Statistical Analysis: A two-sided Student's t-test was used for statistical data evaluation. Experiments were at least repeated twice using $n \geq 3$ images. For descriptive statistics medians, quantiles and standard deviations were calculated.

\section{Acknowledgements}

J.B. and I.P. contributed equally to this work. This work was supported by the Helmholtz Association's Initiative and Networking Fund (grant VH-NG-621) and European Research Council Starting Grant (ERC2013-StG 337077 DropCellArray). The authors were supported by the BIFTM Helmholtz program.

[1] J. W. Costerton, Z. Lewandowski, D. E. Caldwell, D. R. Korber, H. M. Lappin-Scott, Annu. Rev. Microbiol. 1995, 49, 711.

[2] E. Karunakaran, J. Mukherjee, B. Ramalingam, C. A. Biggs, Appl. Microbiol. Biotechnol. 2011, 90, 1869.

[3] W. Characklis, Biofilms 1990, 523.

[4] K. Vacheethasanee, R. E. Marchant, in Handbook of Bacterial Adhesion: Principles, Methods, and Applications, Springer, Totowa, NJ 2000, p. 73-90.

[5] a) I. Banerjee, R. C. Pangule, R. S. Kane, Adv. Mater. 2011, 23, 690; b) R. O. Darouiche, Clin. Infect. Dis. 1999, 29, 1371; c) R. O. Darouiche, N. Engl. J. Med. 2004, 350, 1422; d) D. J. Stickler, Curr. Opin. Infect. Dis. 2000, 13, 389.

[6] P. Gilbert, P. J. Collier, M. Brown, Antimicrob. Agents Chemother. $1990,34,1865$

[7] J. Wimpenny, W. Manz, U. Szewzyk, FEMS Microbiol. Rev. 2000, 24, 661.

[8] a) H. C. Flemming, J. Wingender, Nat. Rev. Microbiol. 2010, 8, 623; b) P. Stoodley, K. Sauer, D. Davies, J. W. Costerton, Annu. Rev. Microbiol. 2002, 56, 187

[9] a) G. Hamilton, Cancer Lett. 1998, 131, 29; b) S.-M. Ong, Z. Zhao, T. Arooz, D. Zhao, S. Zhang, T. Du, M. Wasser, D. van Noort, 
H. Yu, Biomaterials 2010, 31, 1180; c) N. S. Bhise, J. Ribas, V. Manoharan, Y. S. Zhang, A. Polini, S. Massa, M. R. Dokmeci, A. Khademhosseini, J. Controlled Rel. 2014, 190, 82.

[10] a) J. L. Connell, E. T. Ritschdorff, M. Whiteley, J. B. Shear, Proc. Natl. Acad. Sci. USA 2013, 110, 18380; b) S. E. Cowan, D. Liepmann, J. D. Keasling, Biotechnol. Lett. 2001, 23, 1235; c) A. K. Epstein, A. I. Hochbaum, K. Philseok, J. Aizenberg, Nanotechnology 2011, 22, 494007; d) H. Gu, S. Hou, C. Yongyat, S. De Tore, D. Ren, Langmuir 2013, 29, 11145; e) S. Hou, E. A. Burton, R. L. Wu, Y. Y. Luk, D. Ren, Chem. Commun. (Cambridge) 2009, 1207; f) G. M. Kappell, J. P. Grover, T. H. Chrzanowski, Electron. J. Biotechnol. 2009, 12, 10; g) C. Satriano, G. M. L. Messina, S. Carnazza, S. Guglielmino, G. Marletta, Mater. Sci. Eng. 2006, 26, 942.

[11] a) A. K. Epstein, T.-S. Wong, R. A. Belisle, E. M. Boggs, J. Aizenberg, Proc. Natl. Acad. Sci. USA 2012, 109, 13182; b) J. Li, T. Kleintschek, A. Rieder, Y. Cheng, T. Baumbach, U. Obst, T. Schwartz, P. A. Levkin, ACS Appl. Mater. Interfaces 2013, 5, 6704.

[12] M. M. Kendall, V. Sperandio, MBio 2016, 7, e01748.

[13] T.-S. Wong, S. H. Kang, S. K. Tang, E. J. Smythe, B. D. Hatton, A. Grinthal, J. Aizenberg, Nature 2011, 477, 443.

[14] N. Vogel, R. A. Belisle, B. Hatton, T.-S. Wong, J. Aizenberg, Nat. Commun. 2013, 4, 2167.
[15] T. Schwartz, O. Armant, N. Bretschneider, A. Hahn, S. Kirchen, M. Seifert, A. Dotsch, Microb. Biotechnol. 2015, 8, 116.

[16] a) F. L. Geyer, E. Ueda, U. Liebel, N. Grau, P. A. Levkin, Angew. Chem. Int. Ed. 2011, 50, 8424; b) W. Feng, L. Li, E. Ueda, J. Li, S. Heißler, A. Welle, O. Trapp, P. A. Levkin, Adv. Mater. Interfaces 2014, 1, 1400269.

[17] E. Ueda, P. A. Levkin, Adv. Healthcare Mater. 2013, 2, 1425.

[18] a) H.-C. Flemming, in eLS, John Wiley \& Sons, Ltd, 2001; b) K. Sauer, A. K. Camper, G. D. Ehrlich, J. W. Costerton, D. G. Davies, J. Bacteriol. 2002, 184, 1140.

[19] J. Bruchmann, K. Sachsenheimer, B. E. Rapp, T. Schwartz, PLoS One 2015, 10, e0117300.

[20] a) P. Stoodley, R. Cargo, C. J. Rupp, S. Wilson, I. Klapper, J. Ind. Microbiol. Biotechnol. 2002, 29, 361; b) M. C. M. van Loosdrecht, J. J. Heijnen, H. Eberl, J. Kreft, C. Picioreanu, Antonie van Leeuwenhoek 2002, 81, 245; c) P. Stoodley, Z. Lewandowski, J. D. Boyle, H. M. Lappin-Scott, Biotechnol. Bioeng. 1998, 57, 536.

[21] R. E. Hancock, A. M. Carey, J. Bacteriol. 1979, 140, 902.

[22] T. Schwartz, H. Volkmann, S. Kirchen, W. Kohnen, K. Schon-Holz, B. Jansen, U. Obst, FEMS Microbiol. Ecol. 2006, 57, 158.

[23] A. T. Yeung, E. C. Torfs, F. Jamshidi, M. Bains, I. Wiegand, R. E. Hancock, J. Overhage, J. Bacteriol. 2009, 191, 5592. 
Karlsruher Institut für Technologie

\section{Repository KITopen}

Dies ist ein Postprint/begutachtetes Manuskript.

Empfohlene Zitierung:

Bruchmann, J.; Pini, I.; Gill, T. S.; Schwartz, T.; Levkin, P. A.

Patterned SLIPS for the Formation of Arrays of Biofilm Microclusters with Defined Geometries.

2016. Advanced healthcare materials, 6 .

DOI:10.5445/IR/1000062441

Zitierung der Originalveröffentlichung:

Bruchmann, J.; Pini, I.; Gill, T. S.; Schwartz, T.; Levkin, P. A.

Patterned SLIPS for the Formation of Arrays of Biofilm Microclusters with Defined

Geometries.

2016. Advanced healthcare materials, 6 (1), Art.Nr. 1601082.

Doi:10.1002/adhm.201601082 